\title{
The forgotten right ventricle in cardio-oncology
}

\author{
Eiman Jahangir, MD, MPH, ${ }^{\mathrm{a}, \mathrm{b}, \mathrm{c}}$ Matthew E. Harinstein, $M D{ }^{\mathrm{d}}$ \\ Venkatesh L. Murthy, MD, PhD, ${ }^{\mathrm{e}}$ and Javid Moslehi, $\mathrm{MD}^{\mathrm{a}, \mathrm{b}, \mathrm{c}}$ \\ a Division of Cardiovascular Medicine, Department of Medicine, Vanderbilt University Medical \\ Center, Nashville, TN \\ b Division of Oncology, Department of Medicine, Vanderbilt University Medical Center, \\ Nashville, TN \\ c Cardio-Oncology Program, Department of Medicine, Vanderbilt University Medical Center, \\ Nashville, TN \\ d Division of Cardiology, Heart and Vascular Institute, University of Pittsburgh Medical Center, \\ Pittsburgh, PA \\ e Division of Cardiovascular Medicine, Department of Internal Medicine and Frankel Cardiovas- \\ cular Center, University of Michigan, Ann Arbor, MI
}

Received Jan 2, 2019; accepted Jan 3, 2019

doi: $10.1007 / \mathrm{s} 12350-019-01602-5$

See related article, pp. 2154-2163

Advances in breast cancer treatment over the last 30 years have decreased mortality. Systemic adjuvant therapy has been a key factor for this decrease. ${ }^{1} \mathrm{Nev}$ ertheless, cardiotoxicity remains a major concern and a sequelae of chemotherapy, occurring in $4.2 \%$ of all patients treated with adjuvant therapy per a recent study. ${ }^{2}$ Given the explosion of new oncologic therapies which are applied for the treatment of new breast cancer diagnoses each year (estimated to be 250,000 new cases annually in the United States), early detection and treatment of cardiotoxicity are critically important. ${ }^{3}$

Cardiotoxicity occurs in breast cancer due to a number of traditional and novel therapies including anthracyclines, human epidermal growth factor receptor 2 (HER2) inhibitors (e.g., trastuzumab) and radiation. ${ }^{4}$ With anthracyclines and trastuzumab, cardiotoxicity often presents with a reduction in left ventricular ejection fraction (LVEF) prompting frequent screening during cancer therapy and following completion of therapy. Echocardiography is the most commonly used

Reprint requests: Javid Moslehi, MD, Division of Cardiovascular Medicine, Department of Medicine, Vanderbilt University Medical Center, Nashville, TN; javid.moslehi@vanderbilt.edu

J Nucl Cardiol 2020;27:2164-6.

$1071-3581 / \$ 34.00$

Copyright (c) 2019 American Society of Nuclear Cardiology. method for screening due to its accessibility and low cost. Nonetheless, echocardiography primarily screens for overt and subclinical contractile abnormalities while other testing modalities may detect other aspects of early cardiotoxicity including cardiac biomarkers (e.g., troponin) and cardiac magnetic resonance imaging (cMRI). ${ }^{5}$

Metabolic imaging using 18F-fluorodeoxyglucose (FDG) positron emission tomography (PET) is used in oncology to classify malignant versus benign tumors, assist in tumor staging, and monitor response to treatment. In order to quantitate metabolic activity in a tissue, accounting for variation in patient way and administered dose, standardized uptake values (SUVs) may be computed from the FDG activity in a volume of interest. ${ }^{6}$ This metric is commonly used in the evaluation of a wide array of cancers including breast, small cell lung, cervical, and prostate cancers. In general, because a higher SUV reflects greater metabolic activity, higher SUVs are associated with greater likelihood of malignancy. Nonetheless, infectious diseases can also produce high SUVs, ${ }^{7}$ and tissue hypoxia can also cause a shift toward glycolytic metabolism and thus increased SUV. Conversely, low SUVs are more likely to be benign, although small malignant tumors may also produce similar findings. Thus, SUV is seen as an additive though not a definitive tool in determining malignancy. ${ }^{8}$

In cardiology, PET can be used to evaluate myocardial metabolism, inflammation, and ischemia. Several studies have examined cardiac findings on FDG PET images in relation to chemotherapy and radiation 
therapy. Increased left ventricular (LV) uptake has been described among individuals with Hodgkin's Disease treated with anthracyclines. This increase in left ventricular SUV persisted even after completion of therapy, implying possible long-term effects of the anthracycline on the myocardium. ${ }^{9}$ Individuals with low baseline LV SUV more frequently developed cardiotoxicity, suggesting a potential role of FDG-PET to predict cardiotoxicity. Furthermore, increased right ventricular (RV) uptake has been noted on FDG PET studies in cases of pulmonary hypertension. ${ }^{10,11}$ In pulmonary hypertension, RV SUV directly increased with the pulmonary vascular resistance and decreased among those who respond to vasodilator (i.e., epoprostenol) treatment. ${ }^{12}$ Importantly, RV metabolism was correlated with RV workload and strain which may help in identifying early stages of cardiotoxicity.

In this issue of the Journal of Nuclear Cardiology, Kim et al. evaluated changes in myocardial FDG uptakes among 121 individuals with breast cancer both before and after treatment with anthracycline and/or trastuzumab. The authors used baseline and posttherapy tests obtained as part of oncologic treatment and retrospectively evaluated FDG-PET images (and their respective SUVs) focusing on both RV and LV wall uptakes. The authors identified 15 individuals with cardiotoxicity after therapy and compared their results with the remaining individuals. The patients with "cardiotoxicity" had a mean posttherapy LVEF of $44.5 \%$ (compared to $64.9 \%$ in patients with no cardiotoxicity). The presence of RV myocardial uptake (SUV > 1.8), and a change of SUV of the RV myocardial of $>0.4$ were both associated with cardiotoxicity after controlling for age, radiotherapy, and treatment type. Importantly, this correlation between SUV increased uptake did not extend to LV SUV, suggesting that RV SUV was better correlated with cardiotoxicity.

This study has several limitations. First, the authors did not assess RV SUV based on specific cancer therapy. Of the 15 individuals with cardiotoxicity, 7 received anthracycline, 3 received trastuzumab, and 5 received both anthracycline and trastuzumab. Given the different changes in pathophysiology of cardiotoxicity as a result of anthracyclines and HER2 inhibitors, a study where RV FDG-free wall uptake is assessed depending on the individual therapy may reveal different results. Second, the small number of patients evaluated in the current study limits conclusiveness of these results. Third, it is unclear how many patients actually had clinical symptoms and whether the difference in RV SUV correlated with patients with clinical heart failure. Fourth, this was a retrospective study, in which PET scans were performed as part of oncologic monitoring and treatment. The metabolic preparation for these scans was a simple 6-hour fasting rather than a formal inflammation or viability PET preparation. ${ }^{13}$ Consequently, these findings could very well be confounded by diet, insulin resistance, and glycemic state. Further, If FDG-PET imaging were done after initial exposure to therapy, one could assess whether RV SUV is a predictor of cardiomyopathy (which generally occurs later). Conversely, it would be interesting to see if RV SUV normalizes in patients who have reversal of cardiomyopathy following completion of cancer therapy. An earlier study demonstrated that increased LV SUV persisted after completion of anthracycline therapy, but no such research with HER2 receptor inhibitors have been completed. ${ }^{9}$ Finally, it is unclear if the RV SUV increase was in fact a consequence of the increased workload due to the LV dysfunction or a primary metabolic modification due to specific cancer therapy that then resulted in myocardial dysfunction.

Nevertheless, this is an original study that demonstrates RV involvement in chemotherapy-induced cardiotoxicity and presents an additional application of a commonly performed oncologic test. In the evaluation of cardiotoxicity, the RV has been largely forgotten. Most previous imaging research studies in cardio-oncology have focused on the LV size, LVEF, and overall LV metabolism. On the other hand, studies in other cardiovascular disease processes show an important role for the RV. For example, RV FDG uptake correlates with worse outcomes in individuals with dilated cardiomyopathy, indicating that the clinical significance of the RV should not be ignored. ${ }^{14}$ Why increased RV uptake occurs as the LV fails remains unclear. Further research could determine if increased RV metabolism is due to a harder working RV that is compensating for the failing $\mathrm{LV}$, or is it a true sign of a damaged RV that requires a higher metabolic demand. The studies with pulmonary hypertension appear to indicate that the increased metabolism is reversible, though it is unclear if the same is true in cancer therapy-induced cardiomyopathy. ${ }^{12}$

In the future, the utility of PET (and more generally nuclear cardiology) may extend beyond mere cardiotoxicity detection and monitoring and include better mechanistic delineation of cardiotoxicity. Nuclear cardiology may perhaps play an even a bigger role with new, targeted cancer therapies. For example, many new oncologic therapies target cancer metabolism. ${ }^{15}$ FDGPET may better elucidate cardiac metabolic effects of these therapies. In addition, cancer immunotherapies harness and activate the immune system (and specifically $\mathrm{T}$ lymphocytes) and have revolutionized treatment for a number of cancers in recent years. In breast cancer, atezolizumab, an immune checkpoint inhibitor (ICI), prolongs progression-free survival in metastatic triplenegative breast cancer and will soon be approved in 
patients who have been exposed to anthracyclines and radiation. ${ }^{16}$ However, ICIs have been associated with fulminant myocarditis, pericarditis, and vasculitis in a subset of patients, with the increasing reporting of these toxicities given the fast-expanding use of ICIs. ${ }^{17,18}$ Given the difficulty of detecting cardiovascular toxicities in this setting, FDG-PET may serve as an adjunctive imaging modality to detect myocarditis. ${ }^{19}$ In addition, recent development of additional PET radiotracers has dramatically expanded the scope and use of PET to detect endogenous or adoptively transferred immune cells in vitro. ${ }^{20}$ In the future, the use of these "immuno-PET" tracers could be used to identify the specific immune cells that mediate myocarditis and pericarditis among patients with immunotherapy-associated adverse events, potentially enhancing treatment strategies for these conditions. Indeed, we are hopeful that PET may soon become a powerful tool in the arsenal of the cardio-oncologist.

\section{Disclosure}

Eiman Jahangir and Matthew E. Harinstein have nothing to disclose. Venkatesh L. Murthy has minor stock in General Electric and stock options in Ionetix, has received research grants from Siemens and INVIA Medical Imaging Solutions, has served an expert witness for Jubilant Draximage and has served as a consultant for Ionetix and Curium. Javid Moslehi has served as a consultant for Novartis, Pfizer, Bristol-Myers Squibb, Takeda, Regeneron, Ipsen and has received funding from Pfizer and Bristol-Myers Squibb.

\section{References}

1. Berry DA, Cronin KA, Plevritis SK, Fryback DG, Clarke L, Zelen M, Mandelblatt JS, Yakovlev AY, Habbema JD, Feuer EJ. Cancer I and surveillance modeling network C. Effect of screening and adjuvant therapy on mortality from breast cancer. N Engl J Med 2005;353:1784-92.

2. Henry ML, Niu J, Zhang N, Giordano SH, Chavez-MacGregor M. Cardiotoxicity and cardiac monitoring among chemotherapytreated breast cancer patients. JACC Cardiovasc Imaging 2018;11:1084-93.

3. Moslehi JJ. Cardiovascular toxic effects of targeted cancer therapies. N Engl J Med 2016;375:1457-67.

4. Groarke J, Tong D, Khambhati J, Cheng S, Moslehi J. Breast cancer therapies and cardiomyopathy. Med Clin 2012;96:1001-19.

5. Jahangir E, Shah S, Shum K, Baxter C, Fitzpatrick JD, Cole J, Gilliland Y, Polin NM. Risk assessment and management of anthracycline and HER2 receptor inhibitor-induced cardiomyopathy. South Med J 2015;108:71-8.

6. Kinahan PE, Fletcher JW. Positron emission tomography-computed tomography standardized uptake values in clinical practice and assessing response to therapy. Semin Ultrasound CT MR 2010;31:496-505.

7. Ertay T, Sencan Eren M, Karaman M, Oktay G, Durak H. (18)FFDG-PET/CT in initiation and progression of inflammation and infection. Mol Imaging Radionucl Ther 2017;26:47-52.
8. Futamura M, Asano T, Kobayashi K, Morimitsu K, Nawa M, Kanematsu M, Morikawa A, Mori R, Yoshida K. Prediction of macrometastasis in axillary lymph nodes of patients with invasive breast cancer and the utility of the SUV lymph node/tumor ratio using FDG-PET/CT. World J Surg Oncol 2015;13:49.

9. Bauckneht M, Ferrarazzo G, Fiz F, Morbelli S, Sarocchi M, Pastorino F, Ghidella A, Pomposelli E, Miglino M, Ameri P, Emionite L, Ticconi F, Arboscello E, Buschiazzo A, Massimelli EA, Fiordoro S, Borra A, Cossu V, Bozzano A, Ibatici A, Ponzoni M, Spallarossa P, Gallamini A, Bruzzi P, Sambuceti G, Marini C. Doxorubicin effect on myocardial metabolism as a prerequisite for subsequent development of cardiac toxicity: A Translational (18)F-FDG PET/CT observation. J Nucl Med 2017;58:1638-45.

10. Saygin D, Highland KB, Farha S, Park M, Sharp J, Roach EC, Tang WHW, Thomas JD, Erzurum SC, Neumann DR, DiFilippo FP. Metabolic and functional evaluation of the heart and lungs in pulmonary hypertension by gated 2-[18F]-Fluoro-2-deoxy-D-glucose positron emission tomography. Pulm Circ 2017;7:428-38.

11. Can MM, Kaymaz C, Tanboga IH, Tokgoz HC, Canpolat N, Turkyilmaz E, Sonmez K, Ozdemir N. Increased right ventricular glucose metabolism in patients with pulmonary arterial hypertension. Clin Nucl Med 2011;36:743-8.

12. Oikawa M, Kagaya Y, Otani H, Sakuma M, Demachi J, Suzuki J, Takahashi T, Nawata J, Ido T, Watanabe J, Shirato K. Increased [18F]fluorodeoxyglucose accumulation in right ventricular free wall in patients with pulmonary hypertension and the effect of epoprostenol. J Am Coll Cardiol 2005;45:1849-55.

13. Chareonthaitawee P, Beanlands RS, Chen W, Dorbala S, Miller EJ, Murthy VL, Birnie DH, Chen ES, Cooper LT, Tung RH, White ES, Borges-Neto S, Di Carli MF, Gropler RJ, Ruddy TD, Schindler TH, Blankstein R. Joint SNMMI-ASNC expert consensus document on the role of (18)F-FDG PET/CT in cardiac sarcoid detection and therapy monitoring. J Nucl Cardiol 2017;24:1741-58.

14. Wang L, Ma X, Xiang L, Lu M, Yan C, Zhao S, Fang W. The characterization and prognostic significance of right ventricular glucose metabolism in non-ischemic dilated cardiomyopathy. J Nucl Cardiol 2016;23:758-67.

15. Bellinger AM, Arteaga CL, Force T, Humphreys BD, Demetri GD, Druker BJ, Moslehi JJ. Cardio-oncology: How new targeted cancer therapies and precision medicine can inform cardiovascular discovery. Circulation 2015;132:2248-58.

16. Schmid P, Adams S, Rugo HS, Schneeweiss A, Barrios CH, Iwata H, Dieras V, Hegg R, Im SA, Shaw Wright G, Henschel V, Molinero L, Chui SY, Funke R, Husain A, Winer EP, Loi S, Emens LA, Investigators IMT. Atezolizumab and nab-paclitaxel in advanced triple-negative breast cancer. N Engl J Med 2018;379:2108-21.

17. Salem JE, Manouchehri A, Moey M, Lebrun-Vignes B, Bastarache L, Pariente A, Gobert A, Spano JP, Balko JM, Bonaca MP, Roden DM, Johnson DB, Moslehi JJ. Cardiovascular toxicities associated with immune checkpoint inhibitors: An observational, retrospective, pharmacovigilance study. Lancet Oncol 2018;19:1579-89.

18. Moslehi JJ, Salem JE, Sosman JA, Lebrun-Vignes B, Johnson DB. Increased reporting of fatal immune checkpoint inhibitor-associated myocarditis. Lancet 2018;391:933.

19. Nensa F, Kloth J, Tezgah E, Poeppel TD, Heusch P, Goebel J, Nassenstein K, Schlosser T. Feasibility of FDG-PET in myocarditis: Comparison to CMR using integrated PET/MRI. J Nucl Cardiol 2018;25:785-94.

20. McCracken MN, Tavare R, Witte ON, Wu AM. Advances in PET detection of the antitumor $\mathrm{T}$ cell response. Adv Immunol 2016;131:187-231.

Publisher's Note Springer Nature remains neutral with regard to jurisdictional claims in published maps and institutional affiliations. 